\title{
Symmetry Breaking in a Condensate of Light and its Use as a Quantum Sensor
}

\author{
Robert Bennett๑, ${ }^{1,2,{ }^{*}}$ David Steinbrecht, ${ }^{1}$ Yaroslav Gorbachev, ${ }^{1}$ and Stefan Yoshi Buhmann ${ }^{1,2}$ \\ ${ }^{1}$ Physikalisches Institut, Albert-Ludwigs-Universität Freiburg, Hermann-Herder-Str. 4, D-79104 Freiburg im \\ Breisgau, Germany \\ ${ }^{2}$ Freiburg Institute for Advanced Studies (FRIAS), Albertstr. 19, 79104 Freiburg, Germany
}

(Received 5 June 2019; revised manuscript received 10 January 2020; accepted 17 March 2020; published 13 April 2020)

\begin{abstract}
Bose-Einstein condensates (BECs) represent one of the very few manifestations of purely quantum effects on a macroscopic level. Recently, a new type of condensate has been observed - the photon BEC, where light in a dye-filled cavity thermalizes with dye molecules under the influence of an external driving laser, condensing to the lowest-energy mode. Here, we consider medium-induced symmetry breaking in a photon BEC and show that it can be used as a quantum sensor. The introduction of polarizable objects such as chiral molecules lifts the degeneracy between cavity modes of different polarizations. Even a tiny imbalance is imprinted on the condensate polarization, in a "winner-takes-it-all" effect. When used as a sensor for enantiomeric excess, the predicted sensitivity exceeds that of contemporary methods based on circular dichroism. Our results introduce a symmetry-breaking mechanism that is independent of the external pump and demonstrate that the photon BEC can be used for practical purposes.
\end{abstract}

DOI: 10.1103/PhysRevApplied.13.044031

\section{INTRODUCTION}

The pursuit of Bose-Einstein condensates (BECs) has led to continuing innovation across theory and experiment since its first prediction in 1924 [1,2] and experimental observation in 1995 [3]. The vast majority of BECs achieved in the laboratory to date consist of bosonic atoms, which macroscopically populate the ground state once a threshold temperature has been reached. A remarkable recent development has been the photon BEC, the first realization of which in 2010 [4] brought about a flourishing field of modern research (e.g., [5-10]). The condensate of light consists of a macroscopically occupied lowest-energy state of a photon field confined to a cavity, resulting in a central bright spot in the cavity's emission pattern.

In order to obtain a condensate of light, three conditions must be satisfied. The first of these is that photons should be allowed to thermalize in a photon-number-conserving way. As indicated in Fig. 1, this is achieved by filling a cavity with a dye, the emission and absorption spectra of which fulfill the Kennard-Stepanov relation [11,12], which allows the photons to come into thermal contact with the dye medium via repeated absorption and reemission. The second is that an effective photon mass should be provided in order to sustain the analogy with atomic condensates; this is given by the low-frequency cutoff of the cavity mirrors, which causes the photon dispersion relation to be equivalent to that of massive bosons. Finally, a confining

\footnotetext{
*robert.bennett@glasgow.ac.uk
}

potential is needed - this is naturally provided by the curvature of the cavity mirrors. The resulting system is similar to a laser, one crucial difference being that as the pump power is increased there is a sharp jump in the occupation number of the lowest mode, occurring at far below the power required for population inversion.

An aspect of the photon BEC that has not received particular attention, and that uniquely distinguishes it from condensates of massive particles, is its polarization state. Previous experimental investigations have determined that the photon BEC has a much more well-defined polarization than a thermal cloud [13]. This has been investigated in Ref. [14], where it has been shown that the thermalization of the polarization degree of freedom is slow compared to the emission rate of the dye molecules, especially above threshold.

\section{BREAKING SYMMETRY}

In the above-mentioned experimental and theoretical investigations, standing-wave modes of differing polarizations have typically been degenerate in energy, meaning that the polarization degree of freedom is governed entirely by that of the driving laser that seeds the condensate. In this work, we consider a system in which the polarization symmetry is broken. We achieve this here by introducing a medium that supports differently polarized waves with two distinct refractive indices. This lifts the degeneracy between modes of different polarization, so that a nontrivial polarization state emerges as an intrinsic feature of the condensate itself. The difference between the 


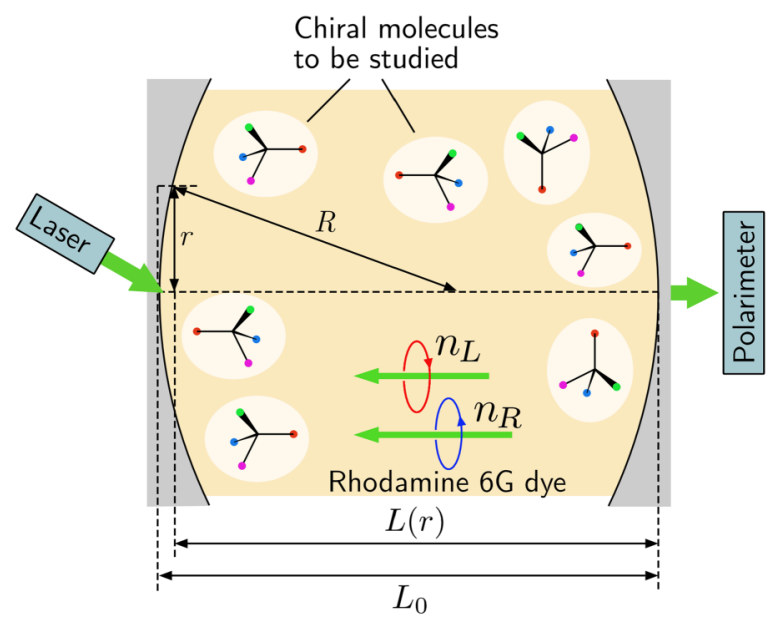

FIG. 1. A schematic of the physical system considered here. The laser pumps the dye molecules and is angled in order to exploit a reflectivity minimum of the plates.

two refractive indices provides a simple degree of freedom to tune, with the resulting emission pattern containing signatures of the nature of the medium inside the cavity.

The symmetry-breaking medium could consist of chiral or anisotropic molecules, or any other system with a polarization-dependent electromagnetic response. The symmetry-broken condensate will then reveal in situ information about this system, such as the handedness, orientation, or conformational state of the molecules. We will use chiral molecules as an example.

We will consider the setup depicted in Fig. 1 where dye molecules fill the space between two gently curved highly reflecting mirrors and are driven by an external laser. The intracavity medium is assumed to be chiral, hence breaking the polarization degeneracy. After determining the energies and loss rates of the polarized photon modes, we extend the model by Keeling and Kirton [5,6], in which a Jaynes-Cummings-type cavity quantum electrodynamics model has been generalized to include the rovibrational states of the dye molecules that are needed to ensure thermalization, to the case of nondegenerate polarization. We give a master equation and rate equations for the coupled light-dye dynamics and use the latter to study the characteristics of the emerging condensate and its dependence on the symmetry-breaking properties of the medium and on the driving laser.

Waves confined in a cavity filled with an optically active medium of chiral susceptibility $\chi$ are subject to different refractive indices $n_{\sigma}=n \pm \chi(\sigma=L, R$; see, e.g., [15]), where $n$ is the refractive index of the intracavity medium, which is assumed to be nonmagnetic $(\mu=1)$. To determine the energies of the circularly polarized standing-wave modes in the cavity, we generalize the procedure given in Ref. [4]. The cavity is assumed to be formed by two curved chiral mirrors [16] of identical radius $R$. Denoting the mirror separation along the optical axis by $L_{0}$, the cavity length at a lateral distance $r$ away from the optical axis is $L(r)=L_{0}-2\left(R-\sqrt{R^{2}-r^{2}}\right)$. Separating the wave vector $k=\sqrt{k_{z}^{2}+k_{\|}^{2}}$ into its normal and lateral components $k_{z}$ and $k_{\|}$, respectively, using the dispersion relation $E=\hbar k c_{\sigma}\left(c_{\sigma}=c / n_{\sigma}\right.$ : the speed of light within the chiral medium, with $c$ denoting the vacuum speed of light) and noting that the normal component of a standing-wave mode has to fulfill the resonance condition $k_{z}=\pi j / L(r)$ $(j=1,2, \ldots)$, the associated energy reads

$$
\begin{aligned}
E_{j \sigma}\left(r, k_{\|}\right) & =\hbar c_{\sigma} \sqrt{\frac{j^{2} \pi^{2}}{L^{2}(r)}+k_{\|}^{2}} \\
& \approx \frac{\pi \hbar c_{\sigma} j}{L_{0}}+\frac{\hbar c_{\sigma} L_{0} k_{\|}^{2}}{2 \pi j}+\frac{\pi \hbar c_{\sigma} j r^{2}}{2 L_{0}^{2} R}
\end{aligned}
$$

in the paraxial approximation $\left(r \ll R, k_{\|} r \ll 1\right)$. The lateral mode structure is hence that of a two-dimensional harmonic oscillator with an effective photon mass $m_{j \sigma}=$ $\pi \hbar j /\left(c_{\sigma} L_{0}\right)$ and a lateral frequency $\omega_{\sigma}^{\|}=c_{\sigma} / \sqrt{L_{0} R}$. A cavity of chiral mirrors that reflect left-handed waves into left-handed ones and similarly for right-handed waves supports standing waves of well-defined circular polarization. Combining this fact with (1), the resulting mode energies

$$
E_{j l \sigma}=\hbar \omega_{j l \sigma}=\hbar \omega_{j \sigma}^{0}+l \hbar \omega_{\sigma}^{\|}
$$

$(l=0,1, \ldots)$ are $(l+1)$-fold degenerate for spherical mirrors where the offset $\hbar \omega_{j \sigma}^{0}=m_{j \sigma} c_{\sigma}^{2}+\hbar \omega_{\sigma}^{\|}$is comprised of the normal-mode energy and the lateral zero-point contribution for the $j$ th standing-wave mode of the cavity, which has an effective photon mass $m_{j \sigma}=\pi \hbar j /\left(c_{\sigma} L_{0}\right)$, where $L_{0}$ is the cavity length as shown in Fig. 1. In the following, we use a multi-index $v=(j, l, \sigma)$ to denote the modes where appropriate.

To describe the dynamics of these modes for imperfectly reflecting mirrors, we also need to determine their cavity leakage or decay constants $\kappa$. To this end, we consider the electromagnetic Green's tensor $\mathbf{G}(\mathbf{r}, \mathbf{r}, \omega)$, which is directly related to the spectral energy density $\rho=$ $(\hbar / \pi)\left(\omega^{2} / c^{2}\right) \operatorname{tr} \operatorname{Im} \mathrm{G}$ of the electromagnetic field inside the cavity. When neglecting the mirror curvature, the cavity Green's tensor is given by

$$
\begin{aligned}
\mathrm{G}^{(1)}(\mathbf{r}, \mathbf{r}, \omega)= & \frac{i}{8 \pi^{2}} \int \frac{d^{2} k_{\|}}{k_{z}} \sum_{\sigma} \frac{1}{D_{\sigma}} \\
& \times\left[r_{\sigma}^{2} e^{2 i k_{z} L_{0}}\left(\mathbf{e}_{\sigma+} \mathbf{e}_{\sigma+}+\mathbf{e}_{\sigma-} \mathbf{e}_{\sigma-}\right)\right. \\
& \left.+r_{\sigma}\left(e^{2 i k_{z} z} \mathbf{e}_{\sigma+} \mathbf{e}_{\sigma-}+e^{-2 i k_{z} z} \mathbf{e}_{\sigma-} \mathbf{e}_{\sigma+}\right)\right]
\end{aligned}
$$


where $r_{\sigma}$ are the reflection coefficients of the cavity mirrors, $\mathbf{e}_{\sigma \pm}$ are the polarization unit vectors for the respective $\sigma$-polarized plane waves traveling in the positive or negative $z$ direction, respectively, and $D_{\sigma}=1-r_{\sigma}^{2} e^{2 i k_{z} L_{0}}$. For two well-reflecting mirrors $\left(\left|r_{\sigma}\right|=1-\delta\right.$ with $\delta \ll$ 1) and in the paraxial approximation, the denominators in the vicinity of the cavity resonances read $D_{\sigma} \propto(\omega-$ $\left.\omega_{j \sigma}^{0}\right)+i \kappa / 2$ with $\kappa=2 \delta c_{\sigma} / L_{0}$, showing that the cavity resonances are of Lorentzian shape.

\section{DYNAMICS}

Generalizing the works of Keeling and Kirton [5,6], the system Hamiltonian can be written in a Jaynes-Cummings [17] form as

$$
\begin{aligned}
\hat{H}= & \sum_{\nu} \hbar \omega_{\nu} \hat{a}_{\nu}^{\dagger} \hat{a}_{v}+\sum_{\nu \alpha} \hbar g_{v}\left(\hat{a}_{\nu} \hat{\sigma}_{\alpha}^{\dagger}+\hat{a}_{\nu}^{\dagger} \hat{\sigma}_{\alpha}\right) \\
& +\sum_{\alpha}\left\{\frac{1}{2} \hbar \omega_{D} \hat{\sigma}_{\alpha}^{z}+\hbar \omega_{V}\left[\hat{b}_{\alpha}^{\dagger} \hat{b}_{\alpha}+\sqrt{S} \hat{\sigma}_{\alpha}^{z}\left(\hat{b}_{\alpha}+\hat{b}_{\alpha}^{\dagger}\right)\right]\right\} .
\end{aligned}
$$

Here, the first term is the Hamiltonian of the intracavity field with mode creation and annihilation operators $\hat{a}_{\nu}^{\dagger}, \hat{a}_{v}$; the second term describes the interaction in the rotatingwave approximation of the cavity modes, with the dye molecules indexed by $\alpha$ with coupling constants $g_{v}=g_{j l}$ for achiral dye molecules; and the last term is the Hamiltonian of the dye molecules with a single electronic transition of frequency $\omega_{D}$ described by the Pauli operators $\hat{\sigma}_{\alpha}, \hat{\sigma}_{\alpha}^{\dagger}$, and $\hat{\sigma}_{\alpha}^{z}$, which is coupled to rovibrational states of splitting $\omega_{V}$ and associated creation and annihilation operators $\hat{b}_{\alpha}^{\dagger}$ and $\hat{b}_{\alpha}$ via a Huang-Rhys factor $S$.

We apply a polaron transformation $\hat{H} \mapsto \hat{U}^{\dagger} \hat{H} \hat{U}$ with [18]

$$
\hat{U}=\exp \left[\sum_{\alpha} \sqrt{S} \hat{\sigma}_{\alpha}^{z}\left(\hat{b}_{\alpha}-\hat{b}_{\alpha}^{\dagger}\right)\right],
$$

after which the Hamiltonian assumes the form

$$
\begin{aligned}
\hat{H}= & \sum_{\nu} \hbar \omega_{\nu} \hat{a}_{\nu}^{\dagger} \hat{a}_{v}+\sum_{\alpha}\left(\frac{1}{2} \hbar \omega_{D} \hat{\sigma}_{\alpha}^{z}+\hbar \omega_{v} \hat{b}_{\alpha}^{\dagger} \hat{b}_{\alpha}\right) \\
& +\sum_{\nu \alpha} \hbar g_{\nu}\left(\hat{a}_{\nu} \hat{\sigma}_{\alpha}^{\dagger} \hat{D}_{\alpha}+\hat{a}_{\nu}^{\dagger} \hat{\sigma}_{\alpha} \hat{D}_{\alpha}^{\dagger}\right) .
\end{aligned}
$$

The first term is the Hamiltonian of the intracavity field with mode creation and annihilation operators $\hat{a}_{\nu}^{\dagger}, \hat{a}_{\nu}$. The second term is the free Hamiltonian for a dye molecule with a single electronic transition (frequency $\omega_{D}$, described by the Pauli operators $\hat{\sigma}_{\alpha}, \hat{\sigma}_{\alpha}^{\dagger}$, and $\hat{\sigma}_{\alpha}^{z}$ ), and a ladder of rovibrational levels (frequency splitting $\omega_{V}$, described by creation and annihilation operators $\hat{b}_{\alpha}^{\dagger}$ and $\hat{b}_{\alpha}$ ). The final term describes the mutual interactions of photons with the electronic and rovibrational transitions through the coupling constants $g_{v}$ and the Huang-Rhys factor $S$. We can then integrate out the rovibrational degrees of freedom together with those field modes that are only weakly coupled to the molecules, to arrive at a master equation $[5,6]$ :

$$
\begin{aligned}
\dot{\hat{\rho}}= & -\frac{i}{\hbar}\left[\hat{H}_{0}, \hat{\rho}\right]-\left(\sum_{\nu} \frac{\kappa}{2} \mathcal{L}\left[\hat{a}_{\nu}\right]\right. \\
& +\sum_{\alpha}\left\{\frac{\gamma_{\uparrow}}{2} \mathcal{L}\left[\hat{\sigma}_{\alpha}^{\dagger}\right]+\frac{\gamma_{\downarrow}}{2} \mathcal{L}\left[\hat{\sigma}_{\alpha}\right]\right\} \\
& \left.+\sum_{\nu \alpha}\left\{\frac{\gamma_{\uparrow v}}{2} \mathcal{L}\left[\hat{a}_{\nu} \hat{\sigma}_{\alpha}^{\dagger}\right]\right\}+\frac{\gamma_{\downarrow v}}{2} \mathcal{L}\left[\hat{a}_{\nu}^{\dagger} \hat{\sigma}_{\alpha}\right]\right) \hat{\rho},
\end{aligned}
$$

where $\hat{H}_{0}$ describes the Hamiltonian dynamics of the electronic dye transition and the remaining cavity modes (which play no further role here). The Lindblad terms $\mathcal{L}[\hat{O}] \hat{\rho}=\left\{\hat{O}^{\dagger} \hat{O}, \hat{\rho}\right\}-2 \hat{O} \hat{\rho} \hat{O}^{\dagger}$ come from pumping of the dye molecules by an external laser $\left(\gamma_{\uparrow}\right)$, spontaneous decay into field modes that are integrated out $\left(\gamma_{\downarrow}\right)$, and stimulated emission $\left(\gamma_{\downarrow v}\right)$ as well as absorption $\left(\gamma_{\uparrow \nu}\right)$ from field modes included in the dynamics.

The rates for the stimulated emission and absorption processes read $\gamma_{\downarrow v}=2 \operatorname{Re} K_{v}\left(\omega_{D}-\omega_{v}\right)$ and $\gamma_{\uparrow \nu}=$ $2 \operatorname{Re} K_{v}\left(\omega_{v}-\omega_{D}\right)$, with

$$
K_{v}(\omega)=g_{v}^{2} \int_{0}^{\infty} d t\left\langle\hat{D}_{\alpha}(t) \hat{D}_{\alpha}(0)\right\rangle e^{-\left(\gamma_{\uparrow}+\gamma_{\downarrow}\right) t / 2} e^{-i \omega t} .
$$

Note that the spatial variation of both the coupling constant $g_{\nu}$ and the rates $\gamma_{\downarrow}, \gamma_{\downarrow \nu}$, and $\gamma_{\uparrow v}$ are neglected, so that these quantities are equal for all dye molecules. Note that the master equation (7) remains valid beyond the simple molecular model of Eq. (4) when considering molecules with multiple manifolds of rovibrational excitations.

The master equation (7) allows for semiclassical rate equations for the mean photon numbers $N_{v}=\left\langle\hat{a}_{\nu}^{\dagger} \hat{a}_{v}\right\rangle$ and the excited-state population of the molecules $p=\left\langle\hat{\sigma}_{\alpha}^{\dagger} \hat{\sigma}_{\alpha}\right\rangle$ to be deduced as

$$
\begin{aligned}
\dot{N}_{\nu} & =-\kappa N_{\nu}-\gamma_{\uparrow v} N_{v} M(1-p)+\gamma_{\downarrow v}\left(N_{v}+1\right) M p, \\
\dot{p} & =-\Gamma_{\downarrow} p_{e}+\Gamma_{\uparrow}(1-p),
\end{aligned}
$$

with total rates

$$
\begin{aligned}
& \Gamma_{\downarrow}=\gamma_{\downarrow}+\sum_{\nu}(l+1)\left(N_{\nu}+1\right) \gamma_{\downarrow}, \\
& \Gamma_{\uparrow}=\gamma_{\uparrow}+\sum_{\nu}(l+1) N_{\nu} \gamma_{\uparrow \nu},
\end{aligned}
$$

where $M$ is the total number of dye molecules. 
The main quantities of interest here are photon numbers in the stationary state of the driven-dissipative system. Through elimination of the molecular degree of freedom in an adiabatic approximation [19], these can be found as the stationary limit of

$$
\dot{N}_{v}=-\kappa N_{v}+M \frac{\gamma_{\downarrow v}\left(N_{v}+1\right) \Gamma_{\uparrow}-\gamma_{\uparrow v} N_{v} \Gamma_{\downarrow}}{\Gamma_{\uparrow}+\Gamma_{\downarrow}} .
$$

Before solving these equations numerically, it is worth discussing the analytical solution that exists in the single-mode case. For a high-quality cavity with $\kappa \ll$ $\gamma_{\uparrow}, \gamma_{\downarrow}, \gamma_{\uparrow v}, \gamma_{\downarrow v}$, this simplifies to

$$
N_{\nu}= \begin{cases}0, & \text { if } \gamma_{\uparrow}<\tau_{\nu}, \\ \frac{M\left(\gamma_{\uparrow} \gamma_{\downarrow \nu}-\gamma_{\downarrow} \gamma_{\uparrow v}\right)}{\kappa\left(\gamma_{\uparrow \nu}+\gamma_{\downarrow v}\right)}, & \text { if } \gamma_{\uparrow} \geq \tau_{\nu} .\end{cases}
$$

which demonstrates the sharp jump in the occupation number at a threshold pump frequency given by $\gamma_{\downarrow} \gamma_{\uparrow \nu} / \gamma_{\downarrow v} \equiv$ $\tau_{\nu}$. It is worth noting that our description of the gain medium by a single number is a simplifying assumption - the model could be extended to take into account nontrivial spatial mode profiles or the molecular angular distribution [14].

\section{NUMERICAL SIMULATIONS}

The steady-state rate equations (11) are solved with a semidynamical approach used in Ref. [19]. The rates of stimulated emission $\gamma_{\downarrow v}$ and absorption $\gamma_{\uparrow v}$ are derived from the spectrum of Rhodamine $6 \mathrm{G}$, which is fitted to the experimental data [20] via the following functions:

$$
\begin{aligned}
& \gamma_{\downarrow \nu}=\frac{\gamma_{\downarrow \nu}^{0} \delta^{2}}{\delta^{2}+\left(-\Omega_{0}+\omega_{\nu}+\Delta \Omega\right)^{2}}, \\
& \gamma_{\uparrow \nu}=\frac{\gamma_{\downarrow \nu}^{0} \delta^{2}}{\delta^{2}+\left(-\Omega_{0}+\omega_{\nu}-\Delta \Omega\right)^{2}},
\end{aligned}
$$

with the parameters listed in Table I. The resulting emission and absorption spectra are shown in Fig. 2, where the $j=7$ manifold is selected, which is assumed throughout this work.

An example result of the numerical simulations are shown in Fig. 3, where a sharp jump in occupation number for the left-polarized mode is observedas the threshold frequency is crossed. Crucially, this jump is not found in the right-polarized mode, meaning that the output signal of the cavity will have a clear left polarization. This is a clearly measurable signature of the vectorial nature of the condensate, which cannot be achieved with BECs of massive particles. The effect can be quantified by investigating
TABLE I. The parameters used in the numerical simulations. The cavity parameters are taken directly from the experiment [4], the solvent and dye spectral parameters are fitted to the measured data $[20,21]$, and the remainder are taken from theoretical work [5] describing the experiment [4].

\begin{tabular}{llll}
\hline \hline \multicolumn{2}{l}{ Dye and solvent parameters } & \multicolumn{2}{c}{ Cavity parameters } \\
\hline$\delta$ & $50 \mathrm{THz}[20]$ & $R$ & $1 \mathrm{~m} \mathrm{[4]}$ \\
$\gamma_{\downarrow}, g_{\nu}$ & $1 \mathrm{GHz}[5]$ & $L_{0}$ & $1.46 \mu \mathrm{m} \mathrm{[4]}$ \\
$\gamma_{\downarrow \nu}^{0}, \gamma_{\uparrow \nu}^{0}$ & $10 \mathrm{~Hz}[20]$ & $M$ & $10^{9}[5]$ \\
$\Delta \Omega$ & $13.13 \mathrm{THz}[20]$ & $\kappa$ & $100 \mathrm{MHz}[5]$ \\
$\Omega_{0}$ & $3456 \mathrm{THz}[20]$ & & $7\left(\left|r_{\sigma}\right|=0.99\right)$ \\
$n$ & $1.34[21]$ & $j$ & $7[4]$ \\
\hline \hline
\end{tabular}

the third Stokes parameter,

$$
S_{3}=\frac{N_{R}-N_{L}}{N_{R}+N_{L}}
$$

which is shown in the inset of Fig. 3. There, the polarization rapidly jumps from $0>S_{3} \gtrsim-0.1$ (slightly leftcircularly polarized), to $S_{3}=-1$ (strongly left-circularly polarized) as the threshold frequency is passed.

Figure 3 shows that the single-noninteracting-mode approximation does not capture the essential physics of the symmetry-breaking effect. This is to be expected, as the single-mode approach neglects transfer of energy between left- and right-circularly polarized modes, which is the mechanism that allows our system to pick out one polarization. Nevertheless, its qualitative behavior can be described by a pinning process, where whichever polarization has a lower condensation threshold as the pump power is increased becomes macroscopically occupied, with the other one staying pinned to whatever occupation it has when the other polarization hits its threshold (even if the pump power is far above its own threshold). This process is

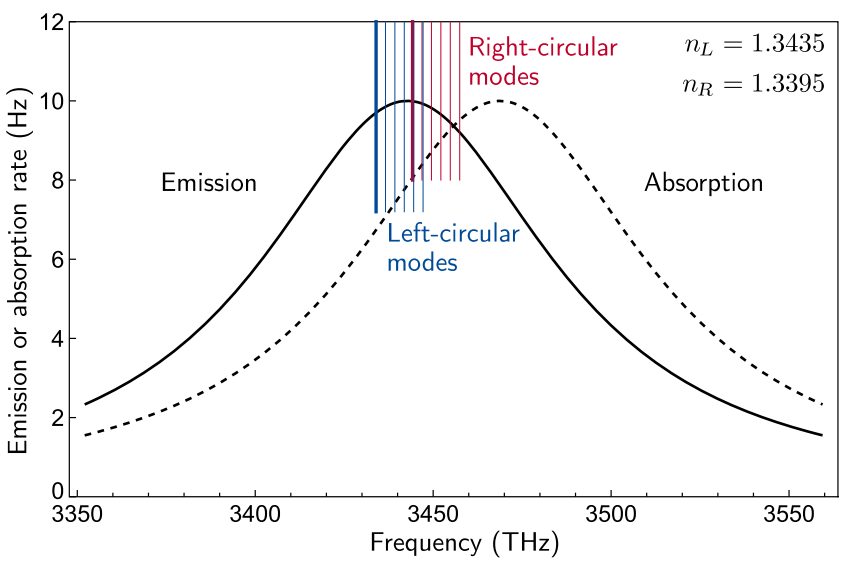

FIG. 2. The emission and absorption spectra. Every tenth mode $(l=0,10,20 \ldots)$ is shown, while the lowest mode for each polarization is shown as a thick line. 


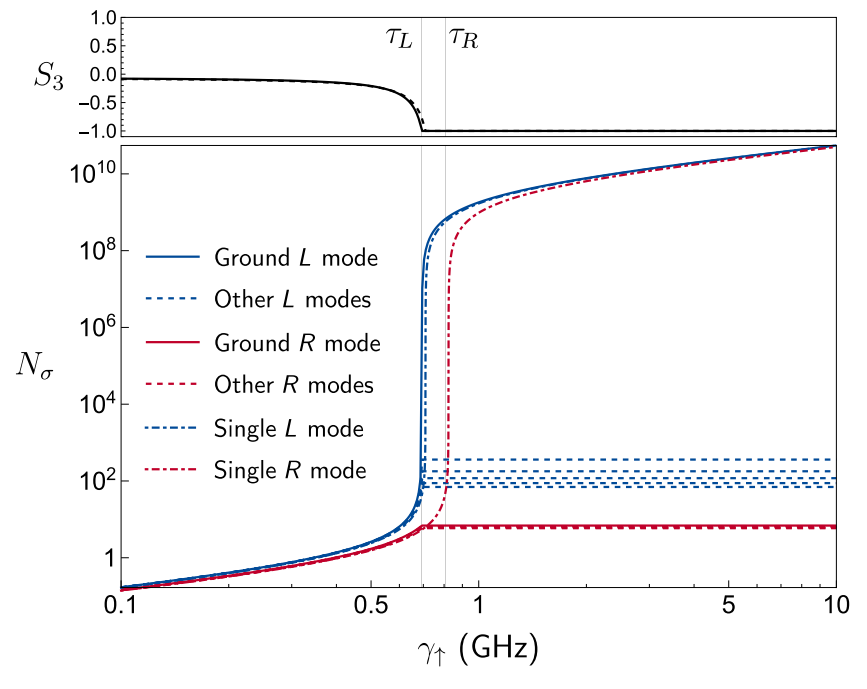

FIG. 3. Polarization symmetry breaking in a photonic BoseEinstein condensate. Lower: the left- and right-mode occupation numbers $N_{L}$ and $N_{R}$ as a function of the pumping rate $\gamma_{\uparrow}$, with refractive indices $n_{L}=1.3435, n_{R}=1.3395$. The inset shows a detail of the threshold region where the "pinning" effect discussed in the text can be observed. Upper: the Stokes parameter $S_{3}$ as a function of the pumping rate $\gamma_{\uparrow}$, using a full numerical approach (solid line) or a single-mode approximation with mode pinning.

the origin of the "winner-takes-it-all" character of the system and, as shown in the upper panel of Fig. 3, this simple approach gives good approximations for the polarization of the output beam. Further physical insight can be gained by using the following formula for the ratio of the occupation $N_{\mathrm{NC}}$ of a given noncondensed mode to the that of the condensed mode $N_{C}$ [22]:

$$
\frac{N_{\mathrm{NC}}}{N_{C}} \approx \frac{2 k_{B} T}{\hbar \Delta N_{\mathrm{tot}}}
$$

where $N_{\text {tot }}$ is the total number of photons. We can use this to predict the number of photons in the lowest rightpolarized mode for the simulations shown in Fig. 3 by taking $N_{\mathrm{NC}}=N_{7,0, R}$ (the lowest right-polarized mode), $N_{C}=N_{7,0, L}, N_{\text {tot }} \approx N_{C}$, and $\Delta=\omega_{7,0, R}-\omega_{7,0, L}$. From this one finds $N_{7,0, R} \approx 8$, in agreement with the simulations shown in 3 . The same can be done for the excited states $n \geq 1$ of the condensed polarization direction by taking $N_{\mathrm{NC}}=N_{7, n, L}$ and $\Delta=\omega_{7,1, L}-\omega_{7, n, L}$, which reproduces the above-threshold occupations shown in Fig. 3.

\section{CHIRAL SENSITIVITY}

Let us assess the ability of the proposed system to detect realistic chiral imbalances. The value of the chiral parameter $\chi$ in terms of the wavelength $\lambda$ of light incident on a sample of molecular number density $\rho$ is [15];

$$
|\chi|=\frac{|\Theta| \alpha \rho m \lambda}{2 \pi} .
$$

Here, $\alpha$ is the volume fraction of chiral molecules of mass $m$ in the whole solution and $\Theta$ is the specific rotation given by

$$
\Theta=\alpha\left(\alpha_{R}-\alpha_{L}\right) \theta \cdot 0.01 \mathrm{~m}^{2} / \mathrm{kg} \quad\left(\left|\alpha_{R}-\alpha_{L}\right|=\epsilon\right),
$$

where $\theta$ is the tabulated value for a particular molecule (often itself referred to as the specific rotation) and $\alpha_{\sigma}$ are the volume fractions of each enantiomer within the chiral component of the solution $\left(\alpha_{L}+\alpha_{R}=1\right)$, so that the absolute value of their difference is the enantiomeric excess $\epsilon$. The angle $\theta$ parametrizes how much a particular chiral molecule rotates an incoming beam of circularly polarized light, independent of the concentration and propagation length. The number density of the methanol that fills the cavity in the proposed device is $\rho=1.488 \times 10^{28} \mathrm{~m}^{-3}$ and its operating frequency of $3450 \mathrm{THz}$ corresponds to a wavelength of $546 \mathrm{~nm}$. Substituting these values into Eq. (16) via Eq. (17), one arrives at

$$
|\chi|=2.14 \times 10^{-8} \theta m_{u} \in \alpha^{2},
$$

where $m_{u}$ is the mass of each chiral molecule in atomic mass units $u=1.66 \times 10^{-27} \mathrm{~kg}, \theta$ is the tabulated specific rotation of the chiral molecules with volume fraction $\alpha$, and $\epsilon$ is the enantiomeric excess, defined as the absolute value of the difference between the volume fractions of the two enantiomers. Precise determination of $\epsilon$ is the subject of considerable pharmacological interest [23]. Considering a solute of glucose (mass $180 u, \theta=44^{\circ}$ ) with volume fraction $\alpha=40 \%$, one finds that $\chi=2.7 \times 10^{-5} \epsilon$. The enantiomeric excess is shown as a percentage on the upper axis of Fig. 4, where $\chi$ is varied for a single pump frequency well above threshold $\left(\gamma_{\uparrow}=10 \mathrm{GHz}\right)$. The gradient $d S_{3} / d \epsilon$ at $\epsilon=0.5$ is approximately 30 , showing that a state-of-the-art cryogenic enantiosensitivity of 0.02 [24] can be detected as a shift of around 0.6 in the Stokes parameter $S_{3}$. The accuracy of the Stokes-parameter measurements already carried out in the first (and so far only) photon BEC polarization experiments [13] was around \pm 0.2 . Figure 4 also demonstrates how the sensitivity of the device and its operating window (i.e., the range and slope of the circular polarization as a function of the enantiomeric excess between the two plateau regions) can be modified by varying the dye-molecule response $\gamma_{\uparrow \nu}$ at the frequency of the laser-cavity system. Finally, Fig. 5 shows a summary of the ideas presented here, where both the refractive-index difference and the pumping strength are varied simultaneously. While the system is unpolarized below threshold, a unique circular polarization emerges 


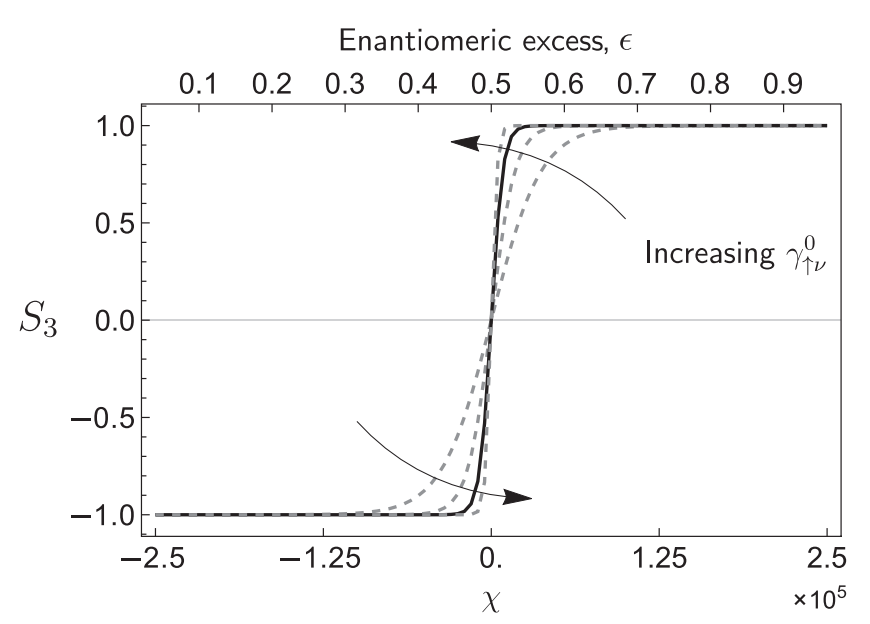

FIG. 4. The use of the photonic Bose-Einstein condensate as a detector for enantiomeric excess. The condensate polarization $S_{3}$ as a function of the refractive-index difference and the chiral parameter $\chi$ (multiplied by $10^{5}$ ) and the enantiomeric excess $\epsilon$. The solid line corresponds to the parameters listed in Table I, while the dotted lines have $\gamma_{\uparrow \nu}^{0}$ scaled by factors of $0.25,0.5$, and 2 in the order indicated.

above threshold that is governed by the enantiomeric excess. We note that any type of chiral molecule or structure that can be dissolved a solvent alongside Rhodamine $6 \mathrm{G}$ could be investigated in this way. Taking methanol as an example solvent as above, these include, for instance, $\alpha$-methyl-2-naphthalenemethanol [25] and phospholipid tubules [26].

Existing methods of chiral discrimination rely on the chiral molecules being in the gas phase and at cryogenic temperatures [24]. The device proposed here allows enantiomeric excess to be detected at room temperature in the liquid phase, offering clear advantages in pharmaceutical and biomedical settings. It could monitor the chirality of a

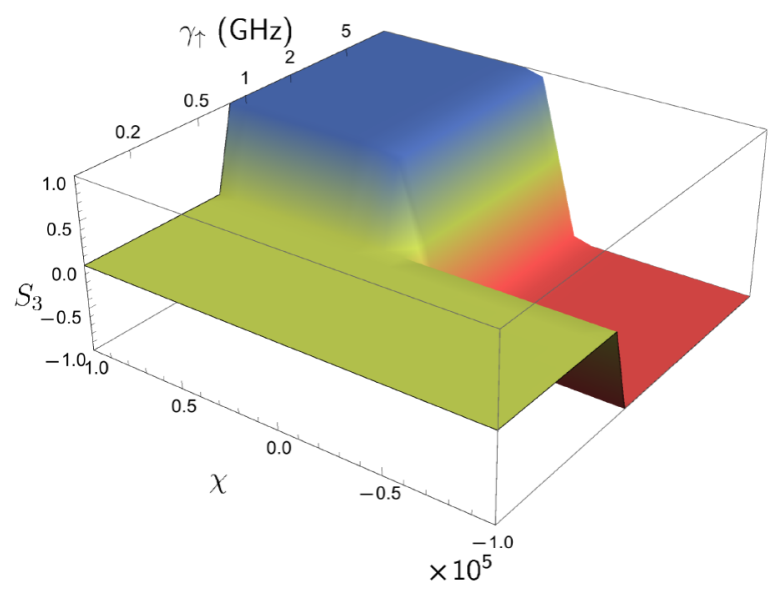

FIG. 5. The Stokes parameter $S_{3}$ as a function of the chiral parameter $\chi$ and the pumping strength $\gamma_{\uparrow}$, using the parameters listed in I. mixture in real time by continuously measuring the polarization of the output light, since the slowest time scale involved in the equilibration process is the inverse cavity decay rate $1 / \kappa=10 \mathrm{~ns}$.

\section{SUMMARY AND CONCLUSIONS}

The photon BEC is a remarkable new physical system, the applications for which are only beginning to be explored. Here, we propose a polarization-symmetry breaking mechanism that can serve as a sensitive probe for magnetoelectric properties of liquid media. We illustrate this with a proof-of-principle calculation for the case of chiral molecules, where the condensate can be used as an extremely sensitive probe of the enantiomeric excess of a liquid medium at room temperature. Our analysis applies to any photon BEC with two different types of mode, such as, for instance, birefringent media, where the horizontal-vertical polarization can serve as a diagnostic tool for detecting the orientation of anisotropic molecules or, by proxy, very weak electric or magnetic fields inducing this orientation. Signatures of conformational chemical reactions could then be observed in real time, having relevance to the emerging field of molecular polaritonics [27], where chemical processes are controlled by an external cavity. A possible future direction to be explored is the interplay of the mode geometry with the spatial distribution of symmetry-breaking probe molecules in analogy with cavity-QED setups for position monitoring and control [28]. In a broader context, this shows that the photon $\mathrm{BEC}$ is ready for an explosion of practical applications in the same way the laser was after its first realization in the laboratory.

\section{ACKNOWLEDGMENTS}

We thank Henry Hesten, Robert Nyman, Andreas Buchleitner, and Akbar Salam for fruitful exchanges. R.B. acknowledges financial support by the Alexander von Humboldt Foundation, Y.G. and S.Y.B. thank the Deutsche Forschungsgemeinschaft (Grant No. BU 1803/3-1476). R.B. and S.Y.B. both acknowledge support from the Freiburg Institute for Advanced Studies (FRIAS).

[1] A. Einstein, Zur Quantentheorie des idealen Gases, Sitzungsberichte der Preußischen Akad. der Wissenschaften 261, 261 (1924).

[2] N. Bose, Plancks Gesetz und Lichtquantenhypothese, Zeitschrift für Phys. 26, 178 (1924).

[3] M. H. Anderson, J. R. Ensher, M. R. Matthews, C. E. Wieman, and E. A. Cornell, Observation of Bose-Einstein condensation in a dilute atomic vapor, Science 269, 198 (1995).

[4] J. Klaers, J. Schmitt, F. Vewinger, and M. Weitz, BoseEinstein condensation of photons in an optical microcavity, Nature 468, 545 (2010). 
[5] P. Kirton and J. Keeling, Nonequilibrium Model of Photon Condensation, Phys. Rev. Lett. 111, 100404 (2013).

[6] P. Kirton and J. Keeling, Thermalization and breakdown of thermalization in photon condensates, Phys. Rev. A 91, 033826 (2015).

[7] E. van der Wurff, A.-W. de Leeuw, R. Duine, and H. Stoof, Interaction Effects on Number Fluctuations in a BoseEinstein Condensate of Light, Phys. Rev. Lett. 113, 135301 (2014).

[8] A.-W. de Leeuw, H. T. C. Stoof, and R. A. Duine, Phase fluctuations and first-order correlation functions of dissipative Bose-Einstein condensates, Phys. Rev. A 89, 053627 (2014).

[9] R. A. Nyman and M. H. Szymańska, Interactions in dyemicrocavity photon condensates and the prospects for their observation, Phys. Rev. A 89, 033844 (2014).

[10] E. Sela, A. Rosch, and V. Fleurov, Condensation of photons coupled to a Dicke field in an optical microcavity, Phys. Rev. A 89, 043844 (2014).

[11] E. H. Kennard, On the thermodynamics of fluorescence, Phys. Rev. 11, 29 (1918).

[12] B. I. Stepanov, A universal relation between the absorption and luminescence spectra of complex molecules, Sov. Phys. Dokl. 2, 81 (1957).

[13] S. Greveling, F. van der Laan, H. C. Jagers, and D. van Oosten, Polarization of a Bose-Einstein condensate of photons in a dye-filled microcavity, arXiv:1712.08426 quant-ph (2017).

[14] R. I. Moodie, P. Kirton, and J. Keeling, Polarization dynamics in a photon Bose-Einstein condensate, Phys. Rev. A 96, 043844 (2017).

[15] M. Schäferling, Chiral Nanophotonics: Chiral Optical Properties of Plasmonic Systems, Springer Series in Optical Sciences (Springer International Publishing, Berlin, 2016).

[16] V. A. Fedotov, A. V. Rogacheva, N. I. Zheludev, P. L. Mladyonov, and S. L. Prosvirnin, Mirror that does not change the phase of reflected waves, Appl. Phys. Lett. 88, 091119 (2006).
[17] E. Jaynes and F. Cummings, Comparison of quantum and semiclassical radiation theories with application to the beam maser, Proc. IEEE 51, 89 (1963).

[18] M. Marthaler, Y. Utsumi, D. S. Golubev, A. Shnirman, and G. Schön, Lasing without Inversion in Circuit Quantum Electrodynamics, Phys. Rev. Lett. 107, 093901 (2011).

[19] H. J. Hesten, R. A. Nyman, and F. Mintert, Decondensation in Nonequilibrium Photonic Condensates: When Less is More, Phys. Rev. Lett. 120, 040601 (2018).

[20] R. A. Nyman, Absorption and fluorescence spectra of Rhodamine 6G [Data set], Zenodo (2017).

[21] C. Sánchez-Pérez and A. García-Valenzuela, Spectroscopic refractometer for transparent and absorbing liquids by reflection of white light near the critical angle, Rev. Sci. Instrum. 83, 115102 (2012).

[22] C. Kurtscheid, D. Dung, E. Busley, F. Vewinger, A. Rosch, and M. Weitz, Thermally condensing photons into a coherently split state of light, Science 366, 894 (2019).

[23] J. Caldwell, N. Berova, and O. McConnell, Chirality in the pharmaceutical industry, Chirality 19, 657 (2007).

[24] D. Patterson and M. Schnell, New studies on molecular chirality in the gas phase: Enantiomer differentiation and determination of enantiomeric excess, Phys. Chem. Chem. Phys. 16, 11114 (2014).

[25] A. R. Al-Rabaa, E. Bréhéret, F. Lahmani, and A. Zehnacker, Enantiodifferentiation in jet-cooled van der Waals complexes of chiral molecules, Chem. Phys. Lett. 237, 480 (1995).

[26] M. S. Spector, K. R. Easwaran, G. Jyothi, J. V. Selinger, A. Singh, and J. M. Schnur, Chiral molecular self-assembly of phospholipid tubules: A circular dichroism study, Proc. Natl. Acad. Sci. U. S. A. 93, 12943 (1996).

[27] J. Feist, J. Galego, and F. J. Garcia-Vidal, Polaritonic chemistry with organic molecules, ACS Photonics 5, 205 (2018).

[28] P. W. H. Pinkse, T. Fischer, P. Maunz, and G. Rempe, Trapping an atom with single photons, Nature 404, 365 (2000). 